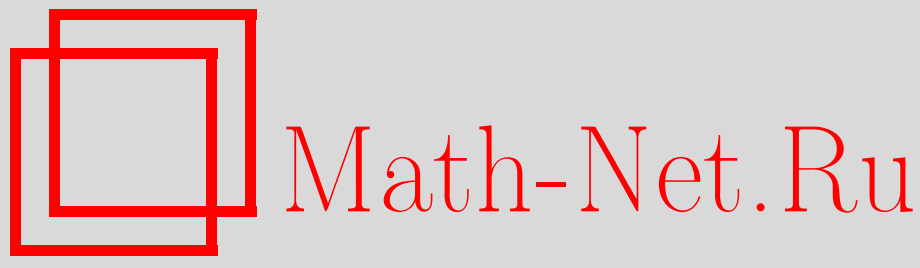

Общероссийский математический портал

В. П. Глушко, Н. А. Ярцева, Об одной эллиптической периодической задаче с вырождением в $L_{p}$, Матем. заметки, 1998, том 63, выпуск 4, 628-632

DOI: https://doi.org/10.4213/mzm1324

Использование Общероссийского математического портала Math-Net.Ru подразумевает, что вы прочитали и согласны с пользовательским соглашением http://www. mathnet.ru/rus/agreement 
Параметры загрузки:

IP : 54.198 .67 .100

26 апреля 2023 г., 03:58:51

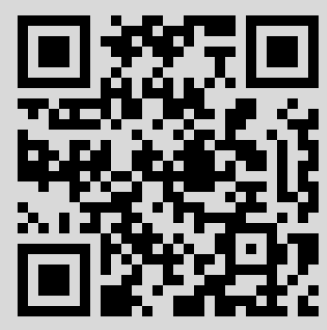




\section{ОБ ОДНОЙ ЭЛЛИПТИЧЕСКОЙ ПЕРИОДИЧЕСКОЙ ЗАДАЧЕ С ВЫРОЖДЕНИЕМ В $L_{p}$}

\section{В.П. Глушко, Н. А. Ярцева}

В работе рассматривается эллиптическое уравнение с существенно переменным коэффициентом по одной переменной $t \in(0, \infty)$, стояшим при производных по этой переменной. Предполагается, что этот коэффициент положителен при всех $t \in(0, \infty)$, слабо вырождается при $t \rightarrow+0$ и достаточно быстро стремится к $\infty$ при $t \rightarrow+\infty$. По остальным переменным $y=\left(y_{1}, y_{2}, \ldots, y_{n-1}\right) \in \mathbb{R}^{n-1}$ предполагается периодичность с периодом $2 \pi$.

Доказывается теорема существования и единственности для соответствующей задачи в $L_{p}\left(\mathbb{R}^{n}\right)$ $(1<p<\infty)$. При построении решения используется специальое весовое преобразование и метод "отражения", позволяющие свести рассматриваемую задачу к периодической по всем переменным. Аналогичный метод использован в работе [1] (см. также [2]).

1. Постановка задачи. В области $G_{n}=T_{0}^{n-1} \otimes \mathbb{R}_{+}$, где

$$
T_{0}^{n-1}=\left\{y \in \mathbb{R}^{n-1}|| y_{j} \mid<\pi, j=\overline{1, n-1}\right\}, \quad \mathbb{R}_{+}=(0, \infty),
$$

рассматривается линейный дифференциальный оператор

$$
L\left(\frac{\partial}{\partial y_{1}}, \frac{\partial}{\partial y_{2}}, \ldots, \frac{\partial}{\partial y_{n-1}}, \alpha(t) \frac{\partial}{\partial t}\right) \equiv L\left(\frac{\partial}{\partial y}, \alpha(t) \frac{\partial}{\partial y}\right)
$$

с однородным степени $m$ эллиптическим символом $L(\xi, \eta), \xi \in \mathbb{R}^{n-1}, \eta \in \mathbb{R}$. Весовая функция $\alpha(t)$ непрерьвно дифференцируема $m-1$ раз и положительна при $t \in \mathbb{R}_{+}$. Точки $t=+0$ и $t=+\infty$ являются в определенном смысле "особыми" для функции $\alpha(t)$, причем эта функция может стремиться к 0 или $\infty$ в указанных точках, однако при этом функция $1 / \alpha(t)$ интегрируема в нуле и существует конечный предел

$$
J(\infty)=\lim _{t \rightarrow+\infty} J(t), \quad J(t)=\int_{0}^{t} \frac{d s}{\alpha(s)} .
$$


В работе изучается уравнение с комплексным параметром $\lambda \in \mathbb{C}$ в классе периодических (c периодом $2 \pi)$ по $y \in \mathbb{R}^{n-1}$ решений:

$$
L\left(\frac{\partial}{\partial y}, \alpha(t) \frac{\partial}{\partial t}\right) u-\lambda u=f(y, t), \quad(y, t) \in G_{n}
$$

при условиях

$$
\lim _{t \rightarrow+0} u(y, t)=\lim _{t \rightarrow+\infty} u(y, t)=0, \quad y \in T_{0}^{n-1} .
$$

Учли, что при $j=0,1, \ldots$

$$
\left(\alpha(t) \frac{\partial}{\partial t}\right)^{j} u=\alpha^{1 / p}(t) D_{\alpha, t, p} u_{\alpha}
$$

где $D_{\alpha, t, p}=\alpha^{1 / p^{\prime}} \frac{\partial}{\partial t} \alpha^{1 / p}(t)\left(1 \leqslant p \leqslant \infty, 1 / p+1 / p^{\prime}=1\right), u_{\alpha}=\alpha^{-1 / p}(t) u$. Мы можем записать задачу $(2),(3)$ в следующем (эквивалентном) виде:

$$
\begin{gathered}
L\left(\frac{\partial}{\partial y}, D_{\alpha, t, p}\right) u_{\alpha}-\lambda u_{\alpha}=f_{\alpha}(y, t), \quad(y, t) \in G_{n} \\
\lim _{t \rightarrow+0} \alpha^{1 / p}(t) u_{\alpha}(y, t)=\lim _{t \rightarrow+\infty} \alpha^{1 / p}(t) u_{\alpha}(y, t)=0, \quad y \in T^{n-1}
\end{gathered}
$$

где $f_{\alpha}(y, t)=\alpha^{-1 / p}(t) f(y, t)$. Решение задачи $(4),(5)$ будем рассматривать в классе $L_{p}^{*} 2 \pi$-периодических по $y \in \mathbb{R}^{n-1}$ функций $u_{\alpha}(y, t)$, интегрируемых по модулю в степени $p(1<p<\infty)$ на любом множестве $T^{n-1} \otimes \mathbb{R}_{+} \subset \mathbb{R}^{n}$, где

$$
T^{n-1}=\left\{y \in \mathbb{R}^{n-1}|| y_{j}-a_{j} \mid \leqslant \pi, j=\overline{1, n-1}\right\} .
$$

В качестве нормы в $L_{p}^{*}$ можно взять

$$
\left\|u_{\alpha}\right\|_{p}=\left(\int_{0}^{\infty} \int_{T_{0}^{n-1}}\left|u_{\alpha}(y, t)\right|^{p} d y d t\right)^{1 / p}
$$

Мы используем также пространство $L_{p, \alpha}^{*}$ функций $u(y, t)$ таких, что $u_{\alpha}(y, t)=\alpha^{-1 / p}(t) u(y, t)$ принадлежит $L_{p}^{*}$. Норму в $L_{p, \alpha}^{*}$ определим равенством

$$
\|u\|_{p, \alpha}=\left\|\alpha^{-1 / p}(t) u\right\|_{p}
$$

К уравнению (4) и условиям (5) применим оператор (см. [2])

$$
G_{\alpha, p}[u(y, t)]=\left.\alpha^{1 / p}(t) u(y, t)\right|_{t=t\left(y_{n}\right)},
$$

где $t=t\left(y_{n}\right)$ - функция, обратная $y_{n}=y_{n}(t)=\gamma J(t)$, а постоянная $\gamma>0$ определяется из условия $\gamma J(\infty)=\pi$.

Учитьвая, что

$$
\begin{gathered}
\left\|G_{\alpha, p}[u]\right\|_{L_{p}\left(T_{0}^{n-1} \otimes(0, \pi)\right)}=\|u\|_{p}, \\
G_{\alpha, p}\left[D_{\alpha, t, p}^{j} u_{\alpha}\right]=\left(\gamma \frac{\partial}{\partial y_{n}}\right)^{j} G_{\alpha, p}\left[u_{\alpha}\right], \quad j=1,2, \ldots,
\end{gathered}
$$

и полагая $v\left(y, y_{n}\right)=G_{\alpha, p}\left[u_{\alpha}(y, t)\right]$, можно записать задачу $(4),(5)$ в виде

$$
\begin{gathered}
L\left(\frac{\partial}{\partial y}, \gamma \frac{\partial}{\partial y_{n}}\right) v-\lambda v=g\left(y, y_{n}\right), \quad y \in T^{n-1}, \quad 0<y_{n}<\pi, \\
\lim _{y_{n} \rightarrow+0} v=\lim _{y_{n} \rightarrow \pi-0} v=0,
\end{gathered}
$$


где $g\left(y, y_{n}\right)=G_{\alpha, p}[f(y, t)]$

Наконец, если обозначить через $\widetilde{v}\left(y, y_{n}\right)$ нечетное продолжение по $y_{n}$ функции $v\left(y, y_{n}\right)$ на $(-\pi, 0)$, то задача $(8)$ может быть сведена к нахождению периодического (с периодом $2 \pi)$ по переменным $\left(y, y_{n}\right)$ решения уравнения

$$
L\left(\frac{\partial}{\partial y}, \gamma \frac{\partial}{\partial y_{n}}\right) \widetilde{v}-\lambda \widetilde{v}=\widetilde{g}\left(y, y_{n}\right), \quad\left(y, y_{n}\right) \in T^{n},
$$

где $\widetilde{g}\left(y, y_{n}\right)$ - нечетное продолжение функции $g\left(y, y_{n}\right)$ на интервал $(-\pi, 0)$. При решении уравнения (9) в классе периодических функций $\widetilde{v}\left(y, y_{n}\right)$ следует иметь в виду, что в силу определения $(6)$ и равенств (7) для любого тора $T^{n}$ имеем

$$
\begin{aligned}
\|\widetilde{v}\|_{L_{p}\left(T^{n}\right)} & =\|\widetilde{v}\|_{L_{p}\left(T_{0}^{n}\right)}=\left(2 \int_{0}^{\pi} \int_{T_{0}^{n-1}}\left|v\left(y, y_{n}\right)\right|^{p} d y d y_{n}\right)^{1 / p} \\
& =2^{1 / p}\left\|u_{\alpha}\right\|_{p}=2^{1 / p}\|u\|_{p, \alpha} .
\end{aligned}
$$

2. Решение периодической задачи. Решение задачи (9) будем искать в виде

$$
\widetilde{v}\left(y, y_{n}\right)=\sum_{\left(l, l_{n}\right)} \widetilde{v}_{l, l_{n}} e^{i\left(l y+l_{n} y_{n}\right)}
$$

где $l y=l_{1} y_{1}+l_{2} y_{2}+\cdots+l_{n-1} y_{n-1}$ и сумма распространяется на все целочисленные мультииндексы $\left(l, l_{n}\right)=\left(l_{1}, l_{2}, \ldots, l_{n-1}, l_{n}\right)$.

Если $\widetilde{g}\left(y, y_{n}\right) \in L_{p}\left(T^{n}\right)$ представить в виде

$$
\widetilde{g}\left(y, y_{n}\right)=\sum_{\left(l, l_{n}\right)} \widetilde{g}_{l, l_{n}} e^{i\left(l y+l_{n} y_{n}\right)},
$$

то для выполнения (9) достаточно потребовать, чтобы

$$
\left(L\left(i l, i \gamma l_{n}\right)-\lambda\right) \widetilde{v}_{l, l_{n}}=\widetilde{g}_{l, l_{n}}, \quad\left|l_{n}\right|>0, \quad \widetilde{v}_{l, 0}=\widetilde{g}_{l, 0}=0 .
$$

Разрешимость (13) основана на вьполнении следующего требования.

УСловие А. При любых $(\xi, \eta) \in \mathbb{R}^{n}$ и $\lambda=\rho e^{i(\theta+m \pi / 2)}, \rho \geqslant 0,|\theta| \leqslant \pi-\varepsilon, \varepsilon>0$, многочлен $L(\xi, \eta)-\lambda$ не обрашается в 0 при $|\xi|^{2}+\eta^{2}+\rho^{2 / m}>0$.

При выполнении условия А система (13) разрешима, поскольку для $\Phi\left(l, l_{n}, \lambda\right)=L\left(i l, i \gamma l_{n}\right)-\lambda=$ $i^{m}\left(L\left(l, \gamma l_{n}\right)-\rho e^{i \theta}\right)$ при $|l|^{2}+l_{n}^{2}+\rho^{2 / m}>0$ справедлива оценка

$$
\left|\Phi\left(l, l_{n}, \lambda\right)\right|^{2}=\left|L\left(l, \gamma l_{n}\right)-\rho e^{i \theta}\right|^{2} \geqslant\left. C_{1}^{2}|| l\right|^{2}+\gamma^{2} l_{n}^{2}+\left.\rho^{2 / m}\right|^{m},
$$

где

$$
C_{1}^{2}=C_{1}^{2}(\theta)=\inf _{|\dot{\xi}|^{2}+\dot{\eta}^{2}+\dot{\rho}^{2 / m}=1}\left|L(\dot{\xi}, \dot{\eta})-\dot{\rho} e^{i \theta}\right|^{2}>0 .
$$

Таким образом, из (11) и (13) находим

$$
\widetilde{v}\left(y, y_{n}\right)=\sum_{\left(l, l_{n}\right)} \frac{\widetilde{g}_{l, l_{n}}}{\Phi\left(l, l_{n}, \lambda\right)} e^{i\left(l y+l_{n} y_{n}\right)} .
$$

Кроме того, из (15) следует

$$
\rho^{\left(m-|\sigma|-\sigma_{n}\right) / m}\left(\frac{\partial}{\partial y}\right)^{\sigma}\left(\gamma \frac{\partial}{\partial y_{n}}\right)^{\sigma_{n}} \widetilde{v}\left(y, y_{n}\right)=\sum_{\substack{\left(l, l_{n}\right) \\\left|l_{n}\right|>0}} \Psi_{\sigma, \sigma_{n}}\left(l, l_{n}, \lambda\right) \widetilde{g}_{l, l_{n}} e^{i\left(l y+l_{n} y_{n}\right)},
$$


где

$$
\Psi_{\sigma, \sigma_{n}}\left(l, l_{n}, \lambda\right)=\frac{\rho^{\left(m-|\sigma|-\sigma_{n}\right) / m} l^{\sigma}\left(\gamma, l_{n}\right)^{\sigma_{n}}}{\Phi\left(l, l_{n}, \lambda\right)} .
$$

Функцию $\Psi_{\sigma, \sigma_{n}}$ можно рассматривать как мульипликатор в пространстве $L_{p}^{*}(1<p<\infty)$ ряда (12) и воспользоваться достаточным условием его ограниченности в $L_{p}^{*}$ (условием Марцинкевича-Михлина, см. [3]), которое в данном случае можно записать в виде: для любых $\alpha_{j}=0$ или 1 , $j=\overline{1, n}$,

$$
\left|\xi_{1}^{\alpha_{1}} \xi_{2}^{\alpha_{2}} \cdots \xi_{n-1}^{\alpha_{n-1}} \eta^{\alpha_{n}} \frac{\partial^{|\alpha|} \Psi_{\sigma, \sigma_{n}}(\xi, \eta, \lambda)}{\partial \xi_{1}^{\alpha_{1}} \partial \xi_{2}^{\alpha_{2}} \cdots \partial \xi_{n-1}^{\alpha_{n-1}} \partial \eta^{\alpha_{n}}}\right| \leqslant C_{\sigma, \sigma_{n}}(\theta)
$$

при всех $(\xi, \eta) \in \mathbb{R}^{n}, \lambda \in \mathbb{C}$, удовлетворяющих условию $\mathrm{A}$.

Проверка условия (16) легко проводится с помощью оценки (14). Таким образом, для любого тора $T^{n}$ при $|\sigma|+\sigma_{n} \leqslant m$ из (16) вытекает

$$
\rho^{\left(m-|\sigma|-\sigma_{n}\right) / m}\left\|\left(\frac{\partial}{\partial y}\right)^{\sigma}\left(\gamma \frac{\partial}{\partial y_{n}}\right)^{\sigma_{n}} \widetilde{v}\right\|_{L_{p}\left(T^{n}\right)} \leqslant K_{p} C_{\sigma, \sigma_{n}}\|\widetilde{g}\|_{L_{p}\left(T_{0}^{n}\right)},
$$

где постоянная $K_{p}>0$ зависит от $p$.

Из (17) следует, что функция $\widetilde{v}\left(y, y_{n}\right)$, определенная формулой (15), принадлежит пространству Соболева $W_{p}^{m}$ в любом торе $T^{n}$ и, следовательно, в любой ограниченной области $\Omega \subset \mathbb{R}^{n}$. Учитывая, что по построению $\widetilde{v}\left(y, y_{n}\right)$ является нечетной функцией $y_{n}$, на основании известных теорем вложения Соболева можно утверждать, что

$$
\lim _{y_{n} \rightarrow+0}\left\|\widetilde{v}\left(\cdot, y_{n}\right)\right\|_{L_{p}\left(T^{n-1}\right)}=\lim _{y_{n} \rightarrow \pi-0}\left\|\widetilde{v}\left(\cdot, y_{n}\right)\right\|_{L_{p}\left(T^{n-1}\right)}=0 .
$$

Тот факт, что $\widetilde{v}\left(y, y_{n}\right)$ является решением уравнения (9), вытекает непосредственно из (13).

\section{3. Существование решения задачи (2), (3). Справедлива следующая}

Tеорема. Пусть $1<p<\infty u \alpha(t)$ удовлетворяет (1). Тогда при выполнении условия А для любой $f(y, t) \in L_{p, \alpha}^{*}\left(G_{n}\right)$ существует единственное решение $u(y, t)$ задачи (2), (3), причем справедлива оченка

$$
\sum_{|\sigma|+\sigma_{n} \leqslant m} \rho^{\left(m-|\sigma|-\sigma_{n}\right) / m}\left\|\left(\frac{\partial}{\partial y}\right)^{\sigma}\left(\alpha(t) \frac{\partial}{\partial t}\right)^{\sigma_{n}} u\right\|_{p, \alpha} \leqslant C\left\|L\left(\frac{\partial}{\partial y}, \alpha(t) \frac{\partial}{\partial t}\right) u-\lambda u\right\|_{p, \alpha},
$$

əде $\lambda=\rho e^{i(\theta+m \pi / 2)}, \rho \geqslant 0,|\theta|<\pi-\varepsilon$. Постоянная $C>0$ зависит лишь от $m, n, p$ и $\theta$.

ДоказАтЕльство. Обозначим через $v\left(y, y_{n}\right)$ сужение функции $\widetilde{v}\left(y, y_{n}\right)$, определенной формулой $(15)$ на $T_{0}^{n-1} \otimes(0, \pi)$. Очевидно, что из (17) немедленно вытекает оценка

$$
\rho^{\left(m-|\sigma|-\sigma_{n}\right) / m}\left\|\left(\frac{\partial}{\partial y}\right)^{\sigma}\left(\gamma \frac{\partial}{\partial t}\right)^{\sigma_{n}} v\right\|_{L_{p}\left(T_{0}^{n-1} \otimes(0, \pi)\right)} \leqslant 2^{1 / p} K_{p} C_{\sigma, \sigma_{n}}\|g\|_{L_{p}\left(T_{0}^{n-1} \otimes(0, \pi)\right)^{\prime}} .
$$

Кроме того, из (18) вытекает

$$
\lim _{y_{n} \rightarrow+0}\left\|v\left(\cdot, y_{n}\right)\right\|_{L_{p}\left(T_{0}^{n-1}\right)}=\lim _{y_{n} \rightarrow \pi-0}\left\|v\left(\cdot, y_{n}\right)\right\|_{L_{p}\left(T_{0}^{n-1}\right)}=0 .
$$

Далее положим $u_{\alpha}(y, t)=G_{\alpha, p}^{-1}\left[v\left(y, y_{n}\right)\right]=\alpha^{-1 / p}(t) v\left(y, y_{n}(t)\right)$. С помощью (7) легко устанавливается, что $u_{\alpha}$ является решением уравнения (4) при $f_{\alpha}=G_{\alpha, p}^{-1}[g]$, а из (21) вытекает справедливость (5). Учитьвая (10), из (20) выводим

$$
\rho^{\left(m-|\sigma|-\sigma_{n}\right) / m}\left\|\left(\frac{\partial}{\partial y}\right)^{\sigma} D_{\alpha, t, p}^{\sigma_{n}} u \alpha\right\|_{L_{p}\left(T_{0}^{n-1} \otimes \mathbb{R}_{+}\right)} \leqslant 2^{1 / p} K_{p} C_{\sigma, \sigma_{n}}\left\|f_{\alpha}\right\|_{L_{p}\left(T_{0}^{n-1} \otimes \mathbb{R}_{+}\right)} .
$$

Поскольку задача (4), (5) эквивалентна задаче (2), (3) для $u(y, t)=\alpha^{1 / p} u_{\alpha}(y, t)$, разрешимость задачи (2), (3) следует из разрешимости задачи (4), (5), а оценка (19) - из оценки (22). 


\section{СПИСОК ЦИТИРОВАННОЙ ЛИТЕРАТУРЫ}

1. Глушко В. П., Малютина О. П. // Тр. матем. фак-та. № 1. Воронеж: Изд-во ВГУ, 1997. С. 29-34. 2. Глушко В. П., Савченко Ю. Б. // Итоги науки и техн. Матем. анализ. Т. 23. М.: ВИНИТИ, 1985. С. 125-218. 3. Трибель Х. Теория интерполяции, функциональные пространства, дифференциальные операторы. М.: Мир, 1980.

Воронежский государственный университет 